\title{
The Maintenance of Sex, Clonal Dynamics, and Host-Parasite Coevolution in a Mixed Population of Sexual and Asexual Snails
}

\author{
Jukka Jokela, ${ }^{1, *}$ Mark F. Dybdahl, ${ }^{2}$ and Curtis M. Lively ${ }^{3}$ \\ 1. EAWAG, Swiss Federal Institute of Aquatic Science and Technology, Überlandstrasse 133, 8600 Dübendorf, Switzerland, and ETH- \\ Zürich, Swiss Federal Institute of Technology Zürich, Institute of Integrative Biology, 8092 Zürich, Switzerland; 2. School of Biological \\ Sciences, Washington State University, Pullman, Washington 99164; 3. Department of Biology, Indiana University, Bloomington, \\ Indiana 47405
}

Aвstract: Sexual populations should be vulnerable to invasion and replacement by ecologically similar asexual females because asexual lineages have higher per capita growth rates. However, as asexual genotypes become common, they may also become disproportionately infected by parasites. The Red Queen hypothesis postulates that high infection rates in the common asexual clones could periodically favor the genetically diverse sexual individuals and promote the short-term coexistence of sexual and asexual populations. Testing this idea requires comparison of competing sexual and asexual lineages that are attacked by natural parasites. To date no such data have been available. Here, we report on long-term dynamics and parasite coevolution in a "mixed" (sexual and asexual) population of snails (Potamopyrgus antipodarum). We found that, within 7-10 years, the most common clones were almost completely replaced by initially rare clones in two different habitats, while sexuals persisted throughout the study period. The common clones, which were initially more resistant to infection, also became more susceptible to infection by sympatric (but not allopatric) parasites over the course of the study. These results are consistent with the Red Queen hypothesis and show that the coevolutionary dynamics predicted by the theory may also favor sexual reproduction in natural populations.

Keywords: coevolution, freshwater snails, maintenance of sexual reproduction, Red Queen hypothesis, Trematoda, parthenogenesis.

\section{Introduction}

The existence of independent segregation of chromosomes accompanied by recombination between maternally and paternally inherited homologues is curious by itself (Otto and Lenormand 2002), but the production of gametes by this mechanism (meiosis) is outright problematic when the gametes are of dramatically different sizes (anisogamy). Anisogamy leads to a cost of sex, whether the gamete types are produced by the same individual (hermaphroditism)

* Corresponding author: e-mail: jukka.jokela@eawag.ch. Am. Nat. 2009. Vol. 174, pp. S43-S53. (C) 2009 by The University of Chicago. 0003-0147/2009/1740\$1-50574\$15.00. All rights reserved. DOI: $10.1086 / 599080$ or by different reproductive morphs (males and females) of the same population (Maynard Smith 1971a, 1978; Williams 1975; Bell 1982). The cost can be ameliorated by factors such as local mate competition and inbreeding, but the cost becomes 1.5-fold for hermaphrodites when investment in male gametes equals the investment in female gametes and twofold for species with separate sexes when the resources invested in sons are equal to the resources invested in daughters (Maynard Smith 1978; Charlesworth 1980; Bell 1982; Lively and Lloyd 1990).

The cost, however, is actually "paid" only when some alternative form of reproduction exists, such as parthenogenesis (the asexual production of genetically identical daughters). When a parthenogen is introduced into the population, it would not produce males, but it would produce twice as many daughters and four times as many granddaughters as the average female in the sexual population (assuming all else is equal). This reproductively isolated clone should rapidly replace the sexual population in the absence of some countervailing force. This is what led John Maynard Smith (1971b) to pose a simple-sounding question in the title of his influential article: what use is sex?

One possibility is that while an asexual mutant might have a reproductive advantage when first introduced into an otherwise sexual population, the advantage is restricted to the situation in which the clone is rare. Suppose the advantage becomes eroded as the clone becomes common, as might be expected when virulent parasites are common, and they infect in a genotype-specific manner. This is the reasoning underlying the Red Queen hypothesis for the advantage to sex (Jaenike 1978; Lloyd 1980; Hamilton 1982; Hamilton et al. 1990). Specifically, parasites might prevent clones with a reproductive advantage from eliminating a diverse sexual population (fig. 1). If so, then parasite-mediated selection could also lead to the short- 

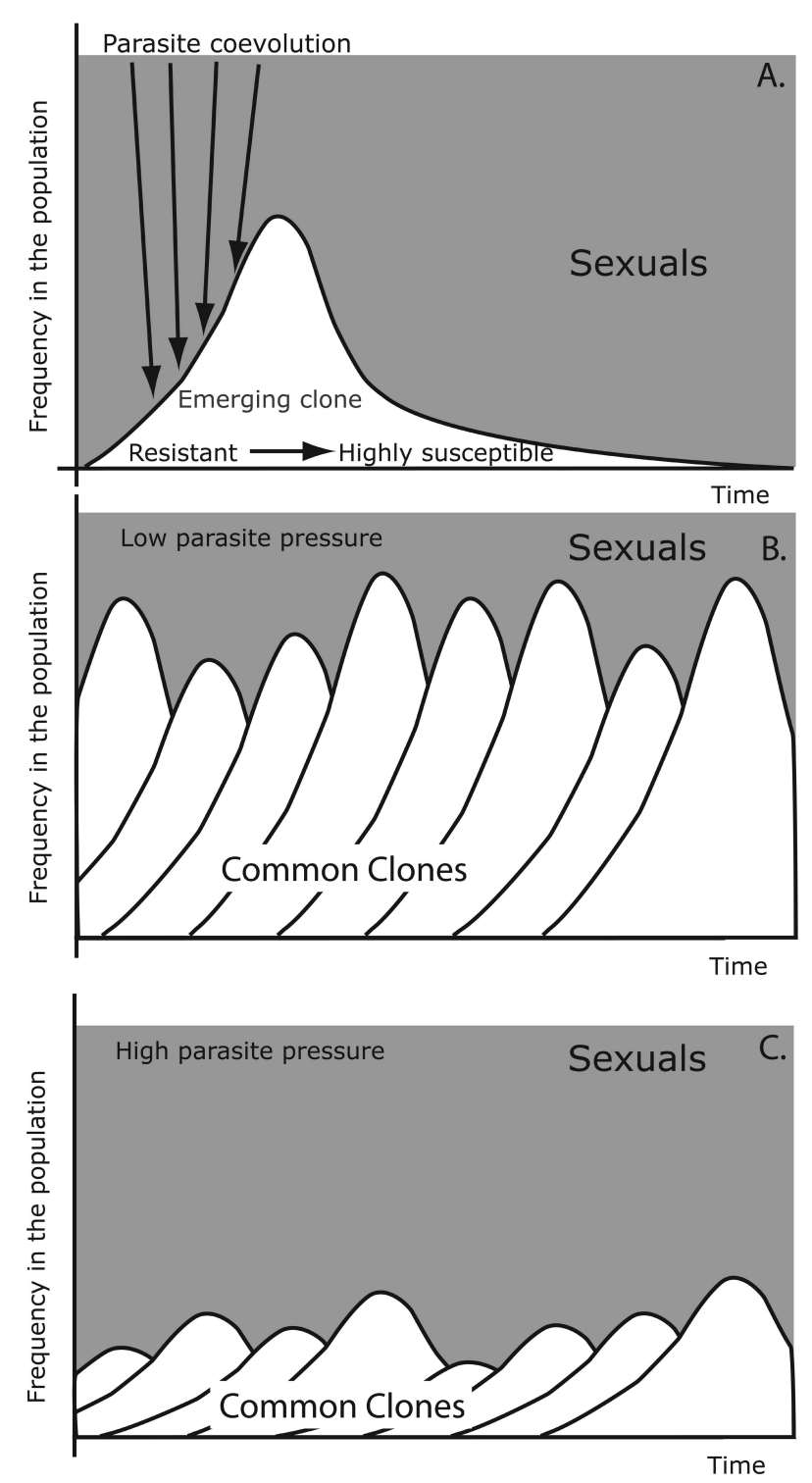

Figure 1: Red Queen rescue of a sexual population. A, Invasion of a high-fitness asexual genotype in a sexual population. In the initial phase, the emerging clone has high fitness (low susceptibility, high growth rate), and it increases in frequency, reducing the relative proportion of the sexual genotypes in the population (gray). As it becomes the most common resistance genotype in the population, natural selection favors parasites that can infect this clone, thus reducing the per capita growth rate of the invading clonal lineage and preventing its further expansion or fixation. $A$ illustrates the Red Queen principle for a single common clone. $B$ and $C$ illustrate a more realistic situation where common clones invade the sexual population in a sequence. Multiple invasion of clones leads to a genetically diverse mixture of sexual and asexual genotypes that is maintained if parasite pressure is strong enough to counter the growth rate advantage of common clones. $B$, Situation where parasite pressure is low (low proportion of sexuals); $C$, situation where parasite pressure is high (high proportion of sexuals). term coexistence of sexual and asexual individuals (Howard and Lively 1994, 1998).

Previous studies have shown that there is parasitemediated selection against common clones within entirely asexual populations of snails and Daphnia (Dybdahl and Lively 1995b, 1998; Little and Ebert 2001; Duncan and Little 2007), and there are data to suggest that such selection can lead to oscillatory dynamics (Dybdahl and Lively 1998; Decaestecker et al. 2007). The next step is to directly examine whether parasite coevolution contributes to the maintenance of sexual reproduction in nature (fig. 1 ), which (assuming a high cost of sex) would require much stronger selection than that required for the maintenance of clonal diversity. Studies of this process require natural host populations in which sexual and asexual individuals coexist in competition for shared resources (we call these populations "mixed").

Mixed populations of sexual and asexual individuals are common in the New Zealand freshwater snail Potamopyrgus antipodarum, which is also the intermediate host for more than a dozen species of digenetic trematodes. In earlier studies, we have shown that sexual and asexual females in mixed populations are ecologically similar and that sexuals pay the cost of sex (Jokela et al. 1997a). We have also shown that local adaptation by the parasites is strong and clone specific and that it may lead to oscillations in the clonal populations (Dybdahl and Lively 1998). We have not, however, directly addressed the Red Queen prediction that selection resulting from parasite coevolution prevents the replacement of sexuals by highly fit clones and thus is a factor promoting the maintenance of sexuals in natural mixed populations. As far as we know, no such data exist for any system.

Using long-term field data combined with laboratory experiments, we examined whether parasites contribute to the evolutionary stability of sexual reproduction in these snails. We found that while the frequency of sexual individuals remained relatively constant over time, the common clonal genotypes changed dramatically. The susceptibility of clones to infection also changed dramatically, such that initially common clones became more susceptible to infection by sympatric parasites.

\section{Material and Methods}

\section{Study System}

Potamopyrgus antipodarum is a prosobranch gastropod that is common in freshwater lakes and streams throughout New Zealand. Some populations are composed of only asexual females, but other populations contain mixtures of sexual and asexual snails (Winterbourn 1970). Earlier work on $P$. antipodarum has suggested that asexual pop- 
ulations prevail in areas associated with a low risk of infection by digenetic trematodes (Lively 1987; Jokela and Lively 1995a; Lively and Jokela 2002), which is consistent with the biogeographical expectations of the Red Queen hypothesis (Glesener and Tilman 1978; Lloyd 1980; Bell 1982).

Genetic studies have shown that asexual snails are triploid, while sexual snails are diploid (Dybdahl and Lively 1995a), but other specific ecological, phenotypic, or lifehistory differences have not been found during several years of study (Jokela et al. 2003). Mixed (sexual and asexual) populations of the snail consist of a variable proportion of sexual individuals and typically contain a highly diverse, locally derived group of triploid, asexual genotypes (Dybdahl and Lively 1995a).

The snail is the first intermediate host to several species of digenetic trematodes, most of which cause complete sterilization of infected snails. An especially common parasite of this snail is a species of Microphallus, which completely sterilizes infected snails (both males and females) and may reach local prevalences exceeding $70 \%$ of the adult population (Jokela and Lively 1995b). Although the worm is not formally described, genetic analyses have shown that the different populations in the central part of the South Island of New Zealand are part of a single widespread species (Dybdahl and Lively 1996). Recent laboratory experiments have shown tight genetic specificity for infection (Dybdahl et al. 2008) and rapid evolution in both the host and the parasite (Koskella and Lively 2007).

\section{Sample Collections}

Each year since 1994, 11-20 sites of the shallow shoreline habitat of Lake Alexandrina (South Island, New Zealand) were visited between mid-January and mid-February (Fox et al. 1996; Jokela et al. 2003). At each site, a large sample of snails (hundreds of individuals) was collected by sweeping vegetation or sediment surface with a kick net. At deeper sites (1-6 m), snorkeling equipment was used to reach the bottom. Samples were transported to Edward Percival Field Station (Kaikoura, New Zealand), where 100 randomly chosen snails were dissected; gender, status of infection, brooding status, and number of eggs were determined for each dissected snail. The 1994 samples were also snap frozen for electrophoresis during dissection (Fox et al. 1996). The material from 1994 represents the reference year for later comparisons. In 2001 and 2003, we sampled the midwater $(1-3 \mathrm{~m})$ and shallow habitats $(<0.5$ $\mathrm{m})$, respectively, for electrophoresis, as in 1994. These snails were brought to the laboratory and later used for infection experiments (in 2001) and genotyped (in 2001 and 2003). The genotype data presented in this study come from these collections and represent the same region of the lake that was sampled in 1994 (the south end of Lake Alexandrina).

\section{Genotyping}

Samples from 1994 were genotyped using six allozyme loci (6PGD, PEP-D, MPI, IDH1, AAT1, and PGM1). We used cellulose acetate gels, on which 24 individuals were run simultaneously. Full description of the protocol is given by Fox et al. (1996). Genotyping for the 2001 infection experiment followed the protocol of 1994. We used three additional loci (AAT2, IDH2, and PGM2) for genotyping the samples of the shallow habitat from 2003.

For identifying the clonal genotypes, we first separated diploids from triploids. We were able to assign ploidy reliably if the individual carried two alleles in any of the loci producing a readable banding pattern. Heterozygous diploids were distinguished from heterozygous triploids on the basis of the asymmetric density of the banding pattern in triploid heterozygotes. In most cases individuals were heterozygous in several loci, improving the already high reliability of the ploidy assignment. The common triploid genotypes were also checked for male frequency, which was very low in all cases. In cases where an individual was homozygous for all loci ( $<10 \%$ of all individuals), ploidy was not assigned, and the individual was not used in the analyses. We could not record a complete genotype for some individuals because electrophoresis produced no readable bands as the result of low-quality samples or lab errors. In cases of missing genotypes for some loci, the multilocus genotype was scored if unambiguous assignment was possible. If not, the individual was not used in any analyses. Unambiguous assignment was not possible for $1.2 \%$ of triploids in 2001 and for $21 \%$ of triploids in 2003. The difference between the years is explained by a freezer failure in 2004 before samples of 2003 were processed. The incomplete genotypes of the rejected triploids of 2003 (43 individuals) were sufficiently detailed to show that they represented a diverse array of unresolved genotypes. It is therefore unlikely that the lower genotyping success of 2003 caused a systematic bias in the results, for example, by specifically focusing on a few common clones. All recognized unique triploid genotypes were assigned a number, and samples from all years, including 1994, were matched to have the same numbering.

After separating the triploid individuals from the diploids, we checked the diploids for common genotypes (potential clones) and found none. We observed no deficiency of homo- or heterozygotes among the diploids in the large sample from 2003 (samples from 10 sites treated as populations, $F_{\text {it }}=0.057$ (low and high $95 \%$ confidence interval: $-0.069,0.222), \quad F_{\text {is }}=0.045 \quad(-0.074,0.205)$, $\left.F_{\mathrm{st}}=0.013(0.002,0.023)\right)$. None of the pairs of loci was 
in statistically significant genotypic disequilibrium (correcting for multiple tests at 5\% risk level; results not shown). The $F$ statistics and linkage tests were conducted with FSTAT, version 2.9.3. These population genetic parameters suggest that the individuals assigned as diploid did not contain common clones and were mainly sexually reproducing individuals.

\section{Infection Experiments}

Potamopyrgus antipodarum is the first intermediate host for several species of trematodes, of which Microphallus sp. is the most common in Lake Alexandrina (Jokela and Lively 1995b). The Potamopyrgus-Microphallus system has been used previously for experimental studies of local parasite adaptation, and the methods of infection experiments are reported in detail in several publications (for references, see Lively et al. 2004). Therefore, only a short summary of the infection protocol of 2001 is given here.

In 2001, we used snails from the midwater (1-3-m deep) habitat as targets for parasites from both Lake Alexandrina (sympatric parasite source) and Lake Poerua (allopatric parasite source); both lakes are located on the South Island of New Zealand. Our prediction was that if parasite coevolution took place, the common genotypes of 1994 would be highly susceptible to sympatric but not to allopatric sources of parasites from 2001. In both lakes, the snails used in the experiment were drawn randomly from a spatially representative set of random samples. On the basis of previous experiments, we expected that Lake Alexandrina parasites would be locally adapted to infect the Lake Alexandrina host population (Lively et al. 2004). Our goal was to have a high infection rate in the sympatric combination (using a high dose) in order to reveal the resistance differences among the common clones. Our second goal was to achieve a sufficient number of infections by the Lake Poerua parasite to test whether variation in resistance among genotypes was dependent on the source of infection.

The experimental infections were carried out in the laboratory (Edward Percival Field Station, Kaikoura, New Zealand), using mice as final hosts. Parasite eggs were obtained from mouse fecal material by repeated dilution of the mouse fecal pellets collected between days 2 and 6 after the mice ingested the cysts. We created three replicate mouse sublines for each of two Microphallus source populations: Lake Alexandrina and Lake Poerua. The Alexandrina sublines were created by feeding each of three mice the metacercariae dissected from 20 infected snails from the Alexandrina population, and the three Poerua mouse sublines were created using 20 infected snails from Lake Poerua. Because the parasites reproduce sexually in the definitive host, the parasite eggs represented recombined genotypes of the 20 parasite genotypes that were give to each mouse.

To expose snails, we added the eggs from each subline to three replicate containers with 100 field-collected snails from Lake Alexandrina. Snails were housed in $2 \mathrm{~L}$ of water with the parasite eggs for 19 days (January 19-February 8 ), with water changed twice per day. The experimental snails were then transported to the United States, where they were held in $2 \mathrm{~L}$ of water, with regular changes of water and food. After about 107 days from initial exposure to parasite eggs, we dissected all surviving snails from each replicate and recorded their infection status. To control for infections that these field-collected snails might have acquired in the field, we also dissected a nonexposed set of control snails. Prevalence of background infections was low and affected all exposure groups similarly. The experimental protocol we used did not allow counting the exact dose of parasite eggs, but on the basis of earlier experience with the system, we expected the dose to be high enough for each host individual to be exposed to several parasite eggs (Osnas and Lively 2004).

\section{Statistical Methods}

We analyzed the results of the infection experiment using a generalized linear model, where infection by Microphallus was used as a binomial response variable (infected or not infected). The independent variables were parasite type (sympatric, allopatric), genotype frequency at present (common 2001, rare 2001), genotype frequency in the past (common 1994, rare 1994), mouse subline (nested within parasite type), replicate container (nested within the mouse subline and parasite type), and snail genotype (partially crossed random effect). We used a logit link function and fitted the model first with all main effects and interactions of the fixed factors. We then dropped interaction terms that did not improve the statistical fit of the model (on the basis of model comparisons using likelihood ratio tests), but we chose to keep all main effects, independent of their statistical significance. We omitted one mouse line from the Lake Alexandrina parasite treatment because the infections failed in the mouse. Clones were pooled on the basis of their frequency so that the 12 most common genotypes (each represented by more than seven individuals in the experiment) were called "common" and the remaining 107 genotypes were called "rare." These common clones covered $75 \%$ of all individuals and $10 \%$ of the genotypes. The choice of 12 clones to represent the common clones was made because it was more conservative than focusing on just a few very common clones. Including more than 12 clones would not have been possible because of the small sample size per clone. Genotypes from 2001 were also classified on the basis of the relative frequency 

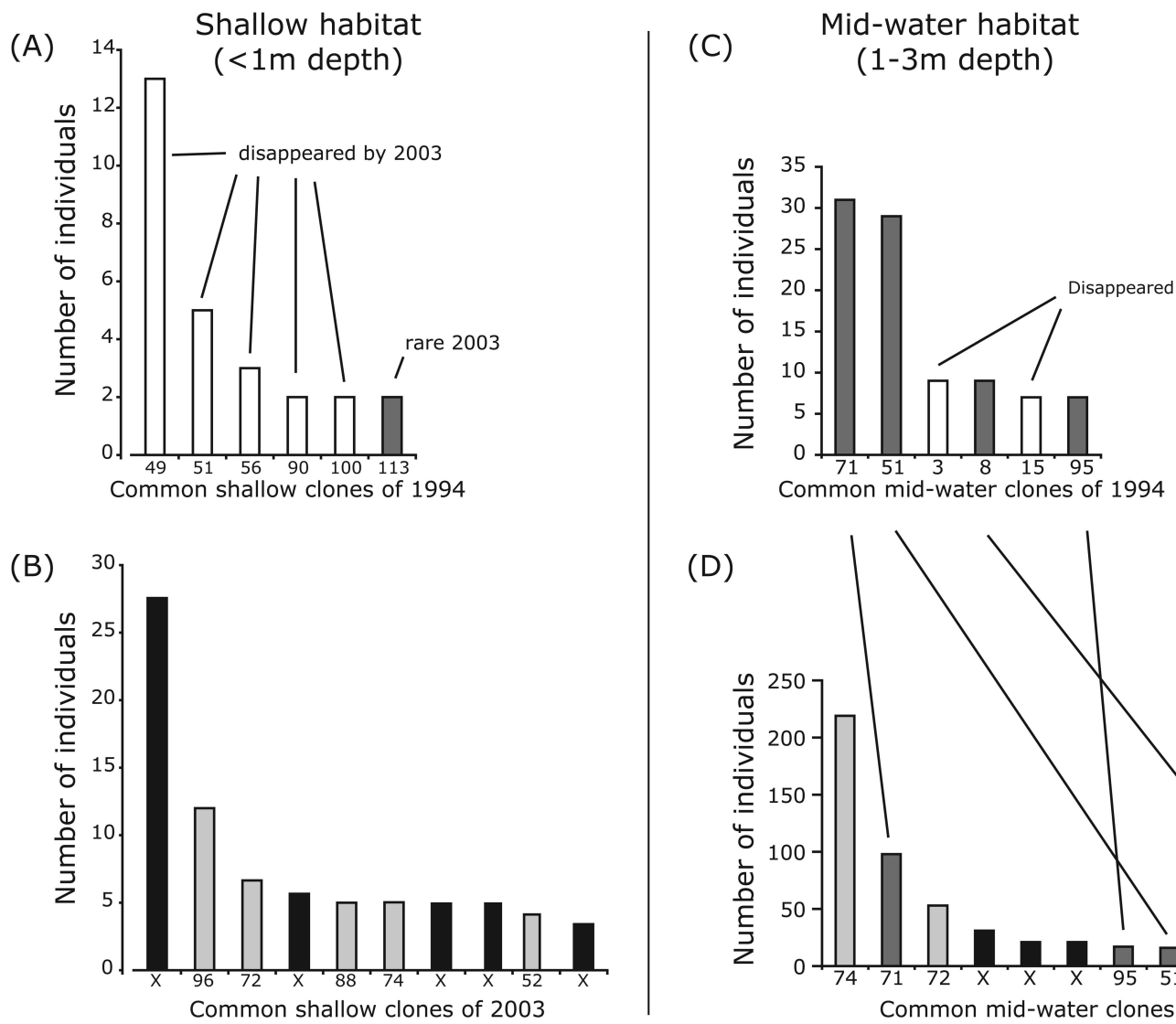

(D)

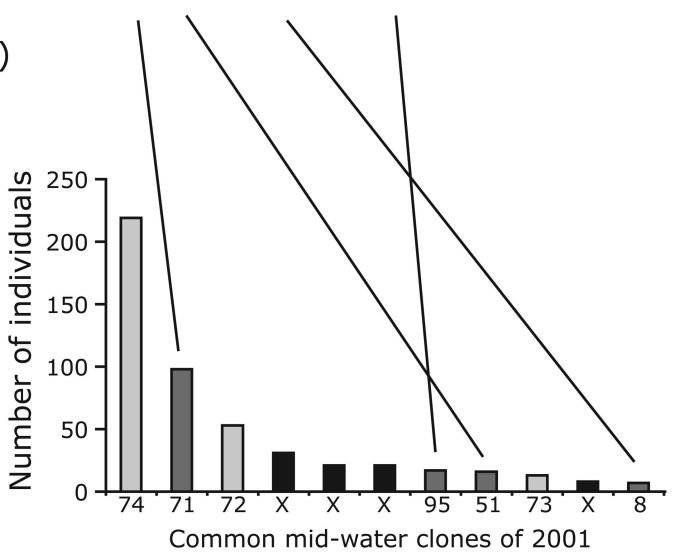

Figure 2: Frequency distributions of the clonal genotypes in shallow and midwater habitats of Lake Alexandrina. A, Common clones of the shallowwater habitat in 1994 (Fox et al. 1996). Shown are genotypes that were found at least twice in a sample of 53 asexual snails. B, Common clones in the shallow-water habitat in 2003. Shown are genotypes that were found at least three times in a sample of 234 asexual snails. $C$, Common clones in the midwater habitat in 1994 (Fox et al. 1996). Shown are genotypes that were found at least seven times in a sample of 197 triploid snails. $D$, Common clones in the midwater habitat in 2001. Shown are genotypes that were found at least seven times in the sample of 682 triploid snails. Numbers on the $X$-axis indicate clonal identity. Black bars indicate genotypes that were not found in 1994 . White bars indicate clones that were not found in 2001 or 2003 but that were found in 1994. Light gray bars indicate clones that were rare in 1994. Dark gray bars indicate clones that were present in 1994 and 2001 (connected by lines to D). In 1994 the two habitats shared a common clone (clone 51).

of the same genotype in the sample of 1994 (Fox et al. 1996). At least one individual of each of the nine most common clones of 1994 was retrieved in 2001, and all of these clones were labeled as "common in the past." The remaining genotypes either were not found or were rare in 1994 and were labeled as "rare in 1994." Because our grouping of clones was post hoc, we were not able to control the genotypic composition of the replicate containers, but we controlled for the container effects statistically. Because individuals of the same genotype can be considered nonindependent, we controlled for the genotype with a random factor. The models were fitted with R package "lme4," using the "glme" function.

\section{Results}

\section{Clonal Dynamics, Mixed Population Structure, and Prevalence of Infection}

We observed a dramatic turnaround in the clonal structure in both habitats. In 1994, a few clones were common and many were rare in both habitats (Fox et al. 1996); the same was true for 2001 and 2003 (for midwater and shallow habitats, respectively; fig. 2). As predicted by the Red Queen hypothesis, the most common clonal genotypes in the shallow habitat declined over the 10-year study period (1994 vs. 2003; see fig. 2A, 2B). Likewise, the most common genotypes of the midwater habitat declined in rank 
or disappeared over the 7-year study period (1994 vs. 2001; fig. $2 C, 2 D$ ).

The observed turnaround of the common clones had no obvious effect on the frequency of sexual individuals in the population. Overall, the frequency of coexisting sexual individuals oscillated over time, but at no point during the course of this study were the sexuals near extinction (fig. 3). Mean male frequency in the shallow habitat over the interval from 1994 to 2003 was $20 \% \pm 7.8 \%$ (mean \pm SE). Similarly, the mean level of infection in the shallow habitat varied between $15.0 \% \pm 4.4 \%( \pm$ SE; 1994$)$ and $25 \% \pm 16.2 \%$ (2003), showing consistently high infection rates during the study, with no obvious response to changes in the clonal structure.

Site-specific examination of male frequency and prevalence of infection revealed considerable differences among the sites as well as variation across the years within sites (fig. 4A). However, none of the sites turned asexual, and most showed rather large changes in frequency of sexuals among the years, emphasizing that the mixed population structure is highly dynamic, even at a local scale (fig. 4A). Site-specific infection patterns corroborate the earlier single-year results of large spatial variation among the sites (Jokela and Lively 1995b) and show that although infection rates vary across the years, some sites seem to have consistently higher infection levels than others (fig. $4 B$ ), which may be explained by patchy distribution of the definitive host of Microphallus parasites (waterfowl).

\section{Infection Experiment to Examine Parasite Coevolution in Common Genotypes}

We found a statistically significant interaction between parasite type (sympatric vs. allopatric) and frequency in 1994 (common or rare; table 1), suggesting that local parasites had adapted to the common host as predicted by the Red Queen theory. We also found a statistically significant interaction between frequency in 1994 and frequency in 2001 (table 1). More specifically, we found that (i) clones that were common in 2001 but that were rare in 1994 were highly resistant to both allopatric parasites and sympatric parasites (group I in fig. 5a), (ii) clones that were common both in 2001 and in 1994 were highly susceptible to sympatric parasites but not to allopatric parasites (group II in fig. $5 a$ ), and (iii) clones that were rare both in 1994 and in 2001 were similarly susceptible to both sympatric parasites and allopatric parasites (group III in fig. $5 a$ ). Clones of group III were also intermediate in susceptibility to sympatric parasites when compared with groups I and II (fig. 5a). The sample size of clones that were rare in 2001 but common in 1994 was too small to draw conclusions about their susceptibility (group IV in fig. $5 a$ ).

We also used this experiment to further examine the prediction that particular common genotypes become more susceptible to sympatric parasites over time. In an earlier study (Jokela et al. 1997b), we reported the prevalence of infection of the five most common clones of

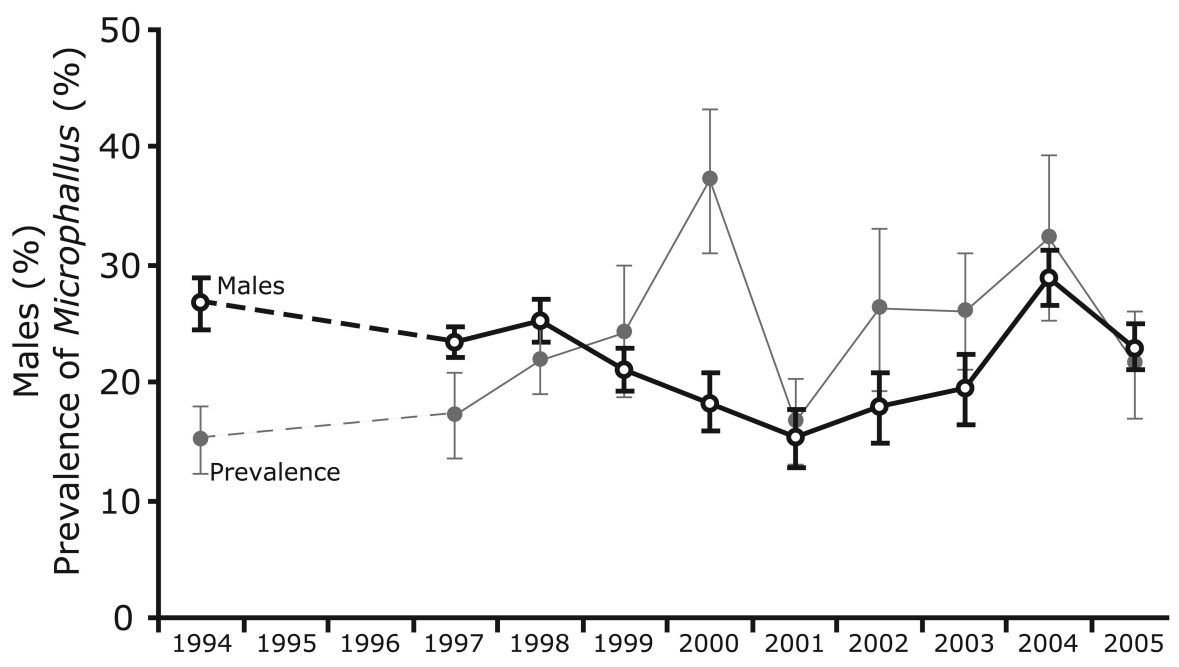

Figure 3: Mean $( \pm$ SE) proportion of sexual snails (male \%) in the shallow-habitat zone of Lake Alexandrina (black) and mean ( \pm SE) prevalence of infection by Microphallus sp. (gray) between 1994 and $2005(n=11$ for all years; same sites sampled each year, and data for years 1995 and 1996 are not available). Proportion of males corresponds closely to proportion of asexual females and can be used as a surrogate of frequency of sexuals in the population (Jokela et al. 2003). Prevalence of infection and male frequency were estimated from random samples of 100 individuals taken annually between mid-January and early February. Site-specific dynamics are shown in figure 4. 

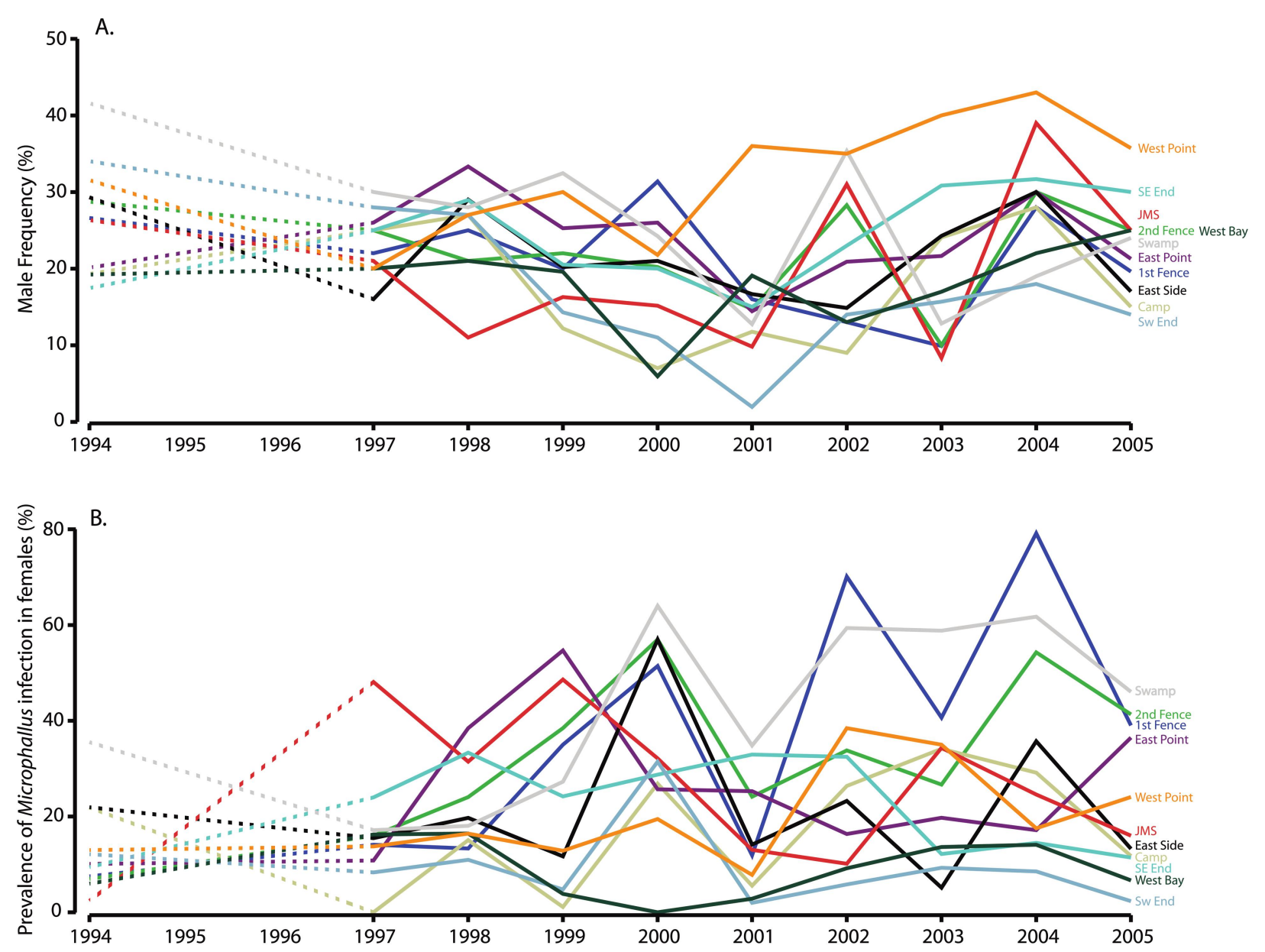

Figure 4: Site-specific dynamics of male frequency $(A)$ and prevalence of Microphallus infection $(B)$ in the shallow-habitat zone of Lake Alexandrina for each of the 11 study sites. Summary of the dynamics is presented in figure 3. Dashed lines connect samples from 1994 to 1997 ; data for 1995 and 1996 are missing. Because the sample size for each site per year was about 100 snails, the $95 \%$ binomial confidence interval for estimates is between $\pm 6 \%$ and $\pm 10 \%$ for values that are between 0.1 and 0.5 .

midwater habitat in 1994. We found that two of the five common clones, clones 51 and 71, were significantly more resistant to Microphallus sp. $\left(\chi^{2}=3.91, \mathrm{df}=1, P=\right.$ .048 and $\chi^{2}=6.13, \mathrm{df}=1, P=.013$ for clones 51 and 71 , respectively) than the sexual snails of the same habitats. Two other clones of the five common ones were overinfected, and one, clone 8 , did not differ from the sexuals (Jokela et al. 1997b).

In 2001, we still found representatives of clones 71, 51, and 8 from the midwater habitat, while the other two overinfected clones of 1994 had disappeared. Clones 51 and 8 had become too rare to evaluate the prevalence of experimental infection in 2001, but clone 71 was still sufficiently represented to make a comparison (fig. $2 D$ ). Analysis of the infection experiment of 2001 showed that Lake Alexandrina parasites were highly infective to clone 71 (fig.
$5 b$ ): $74 \%$ of the individuals were experimentally infected by Microphallus, which is significantly more than the $22 \%$ prevalence of infection that was recorded for the other genotypes $\left(\chi^{2}=32.0, \mathrm{df}=1, P<.001\right)$. In contrast, the Lake Poerua parasites were able to infect $12 \%$ of individuals of clone 71 in the same experiment, which is not statistically different from the $14 \%$ prevalence of infection of the other genotypes in the experiment $\left(\chi^{2}=0.23\right.$, $\mathrm{df}=1, P=.632)$.

\section{Discussion}

The existing empirical data suggest that parasites adapt to their hosts in natural populations (Lively 1989; Ebert 1994; Decaestecker et al. 2007; Wolinska et al. 2007) and that this can lead to selection against common clones in asexual 
Table 1: Statistical analysis of prevalence of infection after experimental inoculations (see also fig. 5)

\begin{tabular}{lrrrrrr}
\hline & Estimate & SD & $Z$ value & $P$ & $\chi^{2}$ & df \\
\hline Fixed effects: & & & & & & \\
$\quad$ Intercept & -4.61 & 1.20 & -3.83 & .0001 & \\
Parasite type & -1.09 & 1.16 & -.940 & .3472 & \\
Frequency at present & 2.13 & .67 & 3.183 & .0015 & \\
Frequency in the past & 5.92 & 1.65 & 3.582 & .0003 & \\
Parasite type $\times$ frequency at present & .33 & .67 & .494 & .6211 & \\
Parasite type $\times$ frequency at past & -1.45 & .67 & -2.187 & .0288 & \\
Frequency at present $\times$ frequency at past & -2.56 & 1.14 & -2.24 & .0250 & & \\
Random effects: & & & & & & \\
Replicate(mouse(parasite type)) & & & & .0249 & 5.029 & 1 \\
Clone(partially crossed random effect) & & & & .0039 & 8.3219 & 1 \\
\hline
\end{tabular}

Note: A random sample of midwater snails of Lake Alexandrina was exposed to sympatric (Lake Alexandrina) and allopatric (Lake Poerua) parasites (fixed effect: parasite type). Snails were genotyped using allozyme electrophoresis and then assigned as common or rare in 1994 (fixed effect: frequency in the past) and common or rare in 2001 (fixed effect: frequency at present). The experiment was replicated by using parasite sublines (random effect: mouse, nested within parasite type) and replicate tanks of each mouse subline (random effect: replicate, nested within mouse and parasite type). Variation due to mouse was included in the effect replicate after the initial model fitting indicated that these two random factors were redundant. Allozyme genotype identity was used to correct for variation among clones (random effect: clone). The fitted model includes all main effects and two-way interactions between fixed factors (log likelihood $=-241$, Aka ke Information Criterion $=500$ ). Analysis was conducted using the generalized mixed model function "glmer," available in package R, for binomial data with a logit link function. Significance of random effects was estimated by comparing the fit of the reduced model to that of the final model, using the "anova" function in R. The test statistics are the difference of $\log$ likel hoods $\left(\chi^{2}\right.$ value) with $1 \mathrm{df}$.

populations, thereby promoting clonal diversity (Dybdahl and Lively 1998; Duncan and Little 2007). While evidence of parasite coevolution selecting for clonal diversity is paramount for understanding the importance of frequencydependent selection by pathogens for maintenance of genetic polymorphism, it does not suffice to argue that the same process is powerful enough to counterbalance the twofold cost of sex in populations where sexual individuals are competing with coexisting clones. Against such costs, selection for the maintenance of sex has to be much stronger than selection for the maintenance of clonal diversity. The outcome of competition between reproductive modes can be addressed only in mixed populations of sexual and asexual individuals.

This study shows that parasite-mediated selection acts against common clones and may prevent an asexual clone from taking over within mixed populations. Our field data revealed surprisingly complete clonal turnover in fewer than 10 years (10-15 generations), indicating strong selection and large differences in susceptibility among the clones. In addition, the proportion of sexual individuals varied during the study but not in a pattern that would indicate a lakewide decrease in proportion of sexuals. However, the analysis based on male frequencies at individual sites within the lake suggests that the frequency of sexuals fluctuates locally (fig. 4). It thus appears that sexual and asexual snails coexist in a dynamic mixture in Lake Alexandrina and that Red Queen coevolution is associated with the demise of common clones that would otherwise have the potential to drive the sexual population to extinction.

Our experimental results also support the hypothesis that emerging clones can be resistant to parasites but become susceptible after becoming common. Results of our infection experiment show that parasites had evolved higher infectivity against the common clonal genotypes of the past. These data also suggest that there was a timelagged association between susceptibility and commonness. Statistically, the strongest significant interaction in the infection experiment was between parasite prevalence and host frequency in the past versus host frequency at present (table 1). Specifically, prevalence of infection of the common clones in the Isoetes (midwater) habitat in 2001 strongly depended on their frequency in 1994 (fig. $5 a)$. Similarly, shallow-water clones that were common in 1994 and still present in 2001 had the highest prevalence of infection (group II in fig. 5a), while those that were rare in 1994 but common in 2001 had the lowest prevalence of infection (group I in fig. 5a). The conclusions at the clonal level are similar. The most common genotype of the past, clone 71 , became highly susceptible to local parasites but not to parasites from an allopatric source population. Hence, we can exclude the possibility that there was a general increase in the susceptibility of clone 71. The greater infectivity in clone 71 most likely resulted from local parasite coevolution.

When infections by sympatric and allopatric parasites are compared, a lower infection rate by allopatric parasites 


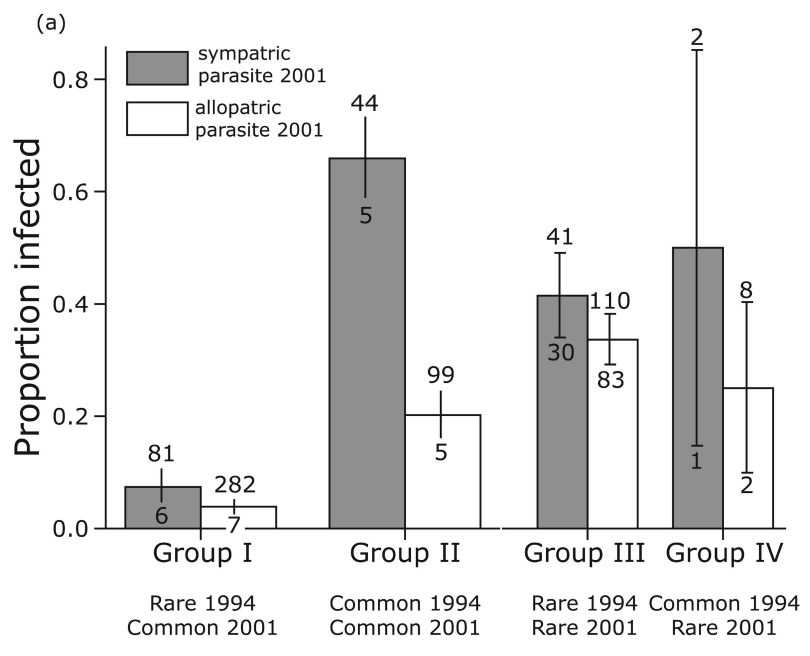

(b)

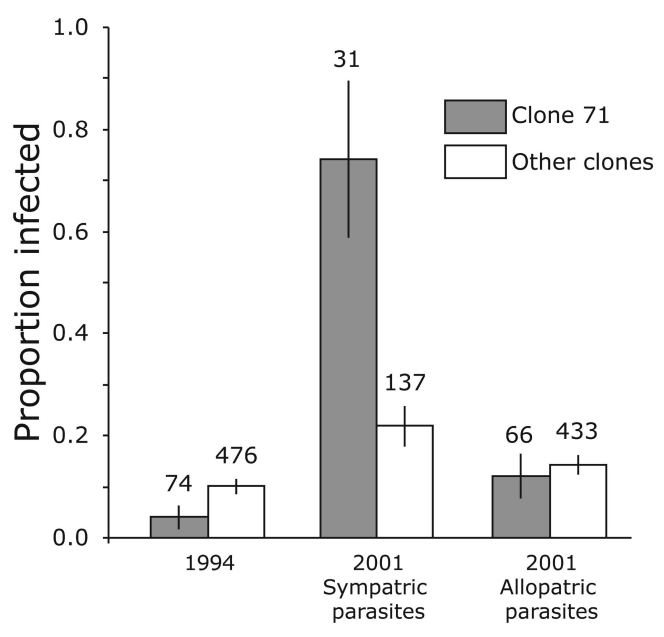

Figure 5: Experimental inoculation of snails from the midwater habitat (1-3 m) of Lake Alexandrina in 2001. a, Proportion of snails infected by the sympatric (Lake Alexandrina) and the allopatric (Lake Poerua) sources of parasites. Snail genotypes are divided into four groups, depending on the frequency of clone in 1994 and 2001 (common vs. rare). Numbers above the error bars indicate the numbers of individuals; numbers below the error bars indicate the numbers of genotypes in 2001. $b$, Prevalence of infection for clone 71 (gray bars), which was the most common clone in 1994, in comparison with other triploid genotypes (white bars). The bars labeled "1994" depict the prevalence of infection in the samples collected in the wild in 1994 (Jokela et al. 1997b; midwater and deep samples pooled). The bars labeled "2001" depict the prevalence of infection in the infection experiment conducted in 2001 (gray bars). Infections by sympatric (Lake Alexandrina) and allopatric (Lake Poerua) parasites are separated. Error bars in both panels are binomial standard errors. Clone 71 is part of group II in $a$.

suggests that sympatric parasites are locally adapted. We have a strong signal of local adaptation in group II (fig. 5), which includes the clones that have remained common since 1994. However, the infection rate by allopatric par- asites seems to vary across the treatment groups, being especially low in group I (rare clones of 1994 that became common by 2001; fig. $5 a$ ). Most of this variation likely reflects variation in the proportion of globally resistant genotypes in the treatment group. Because each group has a different assemblage of genotypes, such variation may be driven by chance events. However, because Microphallus populations experience gene flow (Dybdahl and Lively 1996), it may not be surprising that those host genotypes that are initially resistant to local parasites are initially also resistant to allopatric parasites (group I in fig. 5). It is also possible that some clones are novel genotypes that are resistant to all present parasite genotypes but would become infected by local parasites after a time lag.

The reported dynamics of the clonal populations in the shallow and Isoetes habitats imply that very few of the many rare clones were able to invade the population and become common. In an earlier study focusing on ecological differences among the clones, we found that each common clone expressed a narrow life-history phenotype, possibly reflecting the phenotype of the ancestral sexual genotype (Jokela et al. 1997b). We suspect that these phenotypic differences may translate to fitness differences among the clones and that therefore not all rare clones have equal opportunities to invade the population, independent of parasite resistance. For example, the most common clone in the midwater habitat in 2001 (clone 74), which was also the sixth most common clone of the shallow habitat in 2003, was a rare clone in 1994 (fig. 2). In a parasite-free laboratory common garden of 50 isofemale lines isolated from Lake Alexandrina in 1995, we found that clone 74 , which was isolated twice by chance, had the highest reproductive output of all other isofemale lines, including some of the most common clones of 1994 (Jokela et al. 2003). Yet, clone 74 was a rare clone in 1994, suggesting that in the lake, it had not been able to capitalize its high fitness. This apparent discrepancy was clarified by our present results; we found that clone 74 had become the most common clonal genotype in the lake sometime between 1994 and 2001. Clone 74 was also resistant to parasites in 2001, which, together with the high reproductive fitness, probably aided its success. Future studies will determine whether the fitness of this clone is transitory.

One of the weaknesses of the Red Queen theory for the maintenance of sex has been that very strong parasitemediated selection is required to prevent the fixation of a clone that has the full twofold advantage of asexual reproduction (May and Anderson 1983; Howard and Lively 1994; Otto and Nuismer 2004). The requirement for strong selection would seem to be met in the present study system because the prevalence of infection by trematodes is high in the shallow-water margins of the lake and infection 
invariably sterilizes the snails. The question remains, however, as to whether such strong selection is a general feature for host-parasite interactions in mixed populations of hosts (Lively 2006). The issue can be resolved only by field studies that consider the impact of infection under natural conditions, for which little information exists. However, there are data that show that the impact of infection can be increased under simulated ecological stressors, such as food limitation (Jaenike 1992; Jaenike et al. 1995; Brown et al. 2000, 2003; Tseng 2004; Bedhomme et al. 2005; Jokela et al. 2005), and some data from natural plant populations show that the effect of infection is greater at natural densities than at experimentally reduced host densities (Lively et al. 1995; Bell et al. 2006).

In summary, our results show a dramatic turnover in the clonal structure of a mixed population of sexual and asexual snails. In addition, initially resistant common clones became disproportionately susceptible to infection during the period of clonal turnover. Taken together, the results suggest that parasites might periodically select for sexual reproduction as a consequence of selecting against common clones, as envisioned under the Red Queen hypothesis.

\section{Acknowledgments}

We thank S. Otto for the invitation to participate in this American Society of Naturalists vice presidential symposium. We also thank A. Agrawal, L. Delph, K. Kopp, B. Koskella, Y. Michalakis, S. Otto, K. Räsänen, and J. Wolinska for comments on the earlier versions of the manuscript and the National Science Foundation (C.M.L., J.J., and M.F.D.), the Academy of Finland (J.J.), and the Swiss National Science Foundation (J.J.) for financial support. Finally, we thank the Edward Percival Field Station, especially J. van Berkel and J. McKenzie, for continuous support of our work. Several people have participated in the fieldwork and lab work during the years, and we remain thankful for all of them, especially to A. Alopaeus and J. Fox.

\section{Literature Cited}

Bedhomme, S., P. Agnew, Y. Vital, C. Sidobre, and Y. Michalakis. 2005. Prevalence-dependent costs of parasite virulence. PLoS Biology 3:1403-1408.

Bell, G. 1982. The masterpiece of nature: the evolution and genetics of sexuality. University of California Press, Berkeley.

Bell, T., R. P. Freckleton, and O. T. Lewis. 2006. Plant pathogens drive density-dependent seedling mortality in a tropical tree. Ecology Letters 9:569-574.

Brown, M. J. F., R. Loosli, and P. Schmid-Hempel. 2000. Conditiondependent expression of virulence in a trypanosome infecting bumblebees. Oikos 91:421-427.

Brown, M. J. F., R. Schmid-Hempel, and P. Schmid-Hempel. 2003.
Strong context-dependent virulence in a host-parasite system: reconciling genetic evidence with theory. Journal of Animal Ecology 72:994-1002.

Charlesworth, B. 1980. The cost of sex in relation to mating system. Journal of Theoretical Biology 84:655-671.

Decaestecker, E., S. Gaba, J. A. M. Raeymaekers, R. Stoks, L. V. Kerckhoven, D. Ebert, and L. De Meester. 2007. Host-parasite Red Queen dynamics archived in pond sediment. Nature 450:870-873. Duncan, A. B., and T. J. Little. 2007. Parasite-driven genetic change in a natural population of Daphnia. Evolution 61:796-803.

Dybdahl, M. F., and C. M. Lively. 1995a. Diverse endemic and polyphyletic clones in mixed populations of the freshwater snail, Potamopyrgus antipodarum. Journal of Evolutionary Biology 8:385398.

. 1995b. Host-parasite interactions: infection of common clones in natural populations of a freshwater snail (Potamopyrgus antipodarum). Proceedings of the Royal Society B: Biological Sciences 260:99-103.

- 1996. The geography of coevolution: comparative population structures for a snail and its trematode parasite. Evolution 50:2264-2275.

- 1998. Host-parasite coevolution: evidence for rare advantage and time-lagged selection in a natural population. Evolution 52: 1057-1066.

Dybdahl, M. F., J. Jokela, L. F. Delph, B. Koskella, and C. M. Lively. 2008. Hybrid fitness in a locally adapted parasite. American Naturalist 172:772-782.

Ebert, D. 1994. Virulence and local adaptation of a horizontally transmitted parasite. Science 265:1084-1086.

Fox, J. A., M. F. Dybdahl, J. Jokela, and C. M. Lively. 1996. Genetic structure of coexisting sexual and clonal subpopulations in a freshwater snail (Potamopyrgus antipodarum). Evolution 50:1541-1548.

Glesener, R. R., and D. Tilman. 1978. Sexuality and the components of environmental uncertainty: clues from the geographic parthenogenesis in terrestrial animals. American Naturalist 112:659-673.

Hamilton, W. D., R. Axelrod, and R. Tanese. 1990. Sexual reproduction as an adaptation to resist parasites (a review). Proceedings of the National Academy of Sciences of the USA 87:3566-3573.

Hamilton, W. J. 1982. Pathogens as causes of genetic diversity in their host populations. Pages 269-296 in R. M. Anderson and R. M. May, eds. Population biology of infectious diseases. Springer, New York.

Howard, R. S., and C. M. Lively. 1994. Parasitism, mutation accumulation and the maintenance of sex. Nature 367:554-557.

. 1998. The maintenance of sex by parasitism and mutation accumulation under epistatic fitness functions. Evolution 52:604610.

Jaenike, J. 1978. A hypothesis to account for the maintenance of sex in populations. Evolutionary Theory 3:191-194.

. 1992. Mycophagous Drosophila and their nematode parasites. American Naturalist 139:893-906.

Jaenike, J., H. Benway, and G. Stevens. 1995. Parasite-induced mortality in mycophagous Drosophila. Ecology 76:383-391.

Jokela, J., and C. M. Lively. 1995a. Parasites, sex and early reproduction in a mixed population of freshwater snails. Evolution 49: 1268-1271.

- 1995b. Spatial variation in infection by digenetic trematodes in a population of freshwater snails (Potamopyrgus antipodarum). Oecologia (Berlin) 103:509-517.

Jokela, J., C. M. Lively, M. F. Dybdahl, and J. A. Fox. 1997a. Evidence 
for a cost of sex in the freshwater snail Potamopyrgus antipodarum. Ecology 78:452-460.

. 2003. Genetic variation in sexual and clonal lineages of a freshwater snail. Biological Journal of the Linnean Society 79:165181.

Jokela, J., C. M. Lively, J. A. Fox, and M. F. Dybdahl. 1997b. Flat reaction norms and "frozen" phenotypic variation in clonal snails (Potamopyrgus antipodarum). Evolution 51:1120-1129.

Jokela, J., J. Taskinen, P. Mutikainen, and K. Kopp. 2005. Virulence of parasites in hosts under environmental stress: experiments with anoxia and starvation. Oikos 108:156-164.

Koskella, B., and C. M. Lively. 2007. Advice of the rose: experimental coevolution of a trematode parasite and its snail host. Evolution $61: 152-159$

Little, T. J., and D. Ebert. 2001. Temporal patterns of genetic variation for resistance and infectivity in a Daphnia-microparasite system. Evolution 55:1146-1152.

Lively, C. M. 1987. Evidence from a New Zealand snail for the maintenance of sex by parasitism. Nature 328:519-521.

. 1989. Adaptation by parasitic trematode to local populations of its snail host. Evolution 43:1663-1671.

- 2006. The ecology of virulence. Ecology Letters 9:1089-1095.

Lively, C. M., and J. Jokela. 2002. Temporal and spatial distributions of parasites and sex in a freshwater snail. Evolutionary Ecology Research 4:219-226.

Lively, C. M., and D. G. Lloyd. 1990. The cost of biparental sex under individual selection. American Naturalist 135:489-500.

Lively, C. M., S. G. Johnson, L. F. Delph, and K. Clay. 1995. Thinning reduces the effect of rust infection on jewelweed (Impatiens capensis). Ecology 76:1859-1862.

Lively, C. M., M. F. Dybdahl, J. Jokela, E. E. Osnas, and L. F. Delph. 2004. Host sex and local adaptation by parasites in a snail-trematode interaction. American Naturalist 164(suppl.):S6-S18.
Lloyd, D. G. 1980. Benefits and handicaps of sexual reproduction. Evolutionary Biology 13:69-111.

May, R. M., and R. M. Anderson. 1983. Epidemiology and genetics in the coevolution of parasites and hosts. Proceedings of the Royal Society B: Biological Sciences 219:281-313.

Maynard Smith, J. 1971a. The origin and maintenance of sex. Pages 163-175 in G. C. Williams, ed. Group selection. Aldine, Chicago.

. 1971b. What use is sex? Journal of Theoretical Biology 30: 319-335.

1978. The evolution of sex. Cambridge University Press, Cambridge.

Osnas, E. E., and C. M. Lively. 2004. Parasite dose, prevalence of infection and local adaptation in a host-parasite system. Parasitology 128:223-228.

Otto, S. P., and T. Lenormand. 2002. Resolving the paradox of sex and recombination. Nature Reviews Genetics 3:252-261.

Otto, S. P., and S. L. Nuismer. 2004. Species interactions and the evolution of sex. Science 304:1018-1020.

Tseng, M. 2004. Sex-specific response of a mosquito to parasites and crowding. Proceedings of the Royal Society B: Biological Sciences 271:S186-S188.

Williams, G. C. 1975. Sex and evolution. Princeton University Press, Princeton, NJ.

Winterbourn, M. J. 1970. Population studies on the New Zealand freshwater gastropod Potamopyrgus antipodarum (Gray). Proceedings of the Malacological Society of London 39:139-149.

Wolinska, J., B. Keller, M. Manca, and P. Spaak. 2007. Parasite survey of a Daphnia hybrid complex: host-specificity and environment determine infection. Journal of Animal Ecology 76:191-200.

Symposium Editor: Sarah P. Otto 